\title{
CURVELET-BASED TEXTURE CLASSIFICATION OF TISSUES IN COMPUTED TOMOGRAPHY
}

\author{
Lindsay Semler, Lucia Dettori \\ DePaul University
}

\begin{abstract}
The research presented in this article is aimed at the development of an automated imaging system for classification of tissues in medical images obtained from Computed Tomography (CT) scans. The article focuses on using curvelet-based multi-resolution texture analysis. The approach consists of two steps: automatic extraction of the most discriminative texture features of regions of interest and creation of a classifier that automatically identifies the various tissues. The discriminating power of several curvelet-based texture descriptors are investigated. Tests indicate that Energy, Entropy, Mean and Standard Deviation signatures are the most effective descriptors for curvelets, yielding accuracy rates in the 97 - 98\% range. A comparison with a similar algorithm based on wavelet and ridgelet texture descriptors clearly shows that using curvelet-based texture features significantly improves the classification of normal tissues in CT scans.
\end{abstract}

\section{INTRODUCTION}

The research presented in this article is part of an ongoing project [1] - [3] aimed at developing an automated imaging system for classification of tissues in medical images obtained by Computed Tomography (CT) scans. Classification of human organs in CT scans using shape or gray level information is particularly challenging due to the changing shape of organs in a stack of slices in 3D medical images and the gray level intensity overlap in soft tissues. However, healthy organs are expected to have a consistent texture within tissues across multiple slices. Consequently, the research presented in this article focuses on using texture analysis for the classification of tissues. Texture is a commonly used feature in the analysis and interpretation of images. One way of characterizing texture is by calculating a set of local statistical properties of the pixel grey level intensity, measuring variations in a surface such as smoothness, coarseness and regularity. Traditionally texture features have been calculated using a variety of image processing techniques including run-length statistics [10], cooccurrence matrices [11], statistical moments, fractal dimensions, Gabor filtering, and the wavelet transform [2].

Following the recent introduction of the ridgelet transform, the authors proposed a classification algorithm, which uses ridgelet-based texture features [3]. This research is extended to include texture features based on the discrete curvelet transform [8]. The approach consists of two steps: automatic extraction of the most discriminative curvelet-based texture features of regions of interest and creation of a decision tree classifier that automatically identifies the various tissues. Multiresolution analysis has been successfully used in image processing, and a number of applications to texture classification have been proposed over the past few years [4]. Several studies have investigated the discriminating power of wavelet-based texture features applied to various fields. More recently, applications of the ridgelet transform to image contrast enhancement and image denoising have been explored, as well as applications to texture classification [5]. To the authors' knowledge, curvelet-based texture analysis has been applied only in the context of image representation of astronomical images [6]. This research focuses on texture classification using features derived from the curvelet transform of the images.

Multi-resolution analysis allows for the preservation of an image according to certain levels of resolution or blurring. Broadly speaking, multi-resolution analysis allows for the zooming in and out of the underlying texture structure. Therefore, the texture extraction is not effected by the size of the pixel neighbourhood. This multi-resolution quality is why wavelets have been useful in image compression, image de-noising, and image classification. Wavelets have been an area of research in many texture classification applications [4] and have been useful in capturing texture information and edge detection in natural images, such as detecting the vertical outline of a skyscraper.

By decomposing the image into a series of high-pass and low-pass bands, the wavelet transform extracts directional details that capture horizontal, vertical and diagonal activity. However, these three linear directions are limiting and might not capture enough directional information in noisy images, such as medical CT scans 
which do not have strong horizontal, vertical, and diagonal directional elements. Ridgelets, like wavelets, provide multi-resolution texture information; however they capture structural information of an image based on multiple radial directions in the frequency domain. The authors show in [3] that the multi-directional capabilities of the ridgelet transform provide better texture discrimination than its wavelet counterpart. However, one of the limitations of this approach is the fact that ridgelets are most effective in detecting linear radial structures, which are also not dominant in medical images. A recent extension of ridgelet is the curvelet transform; curve lets are proven to be particularly effective at detecting image activity along curves instead of radial directions. Curve lets also capture structural information along multiple scales, locations, and orientations. Instead of capturing structural information along radial lines, the Curvelet transform captures this structural activity along radial 'wedges' in the frequency domain.

The structural activity extracted from the curvelet transform of the image can be analyzed statistically to generate texture features used in the classifier to create classification rules. Common statistical measures used in texture classification in image processing are: mean, standard deviation, energy, entropy, contrast, homogeneity, variance, correlation, maximum probability, sum-mean, cluster tendency, and inverse difference moment [9]. However, since these statistics are being applied to the curvelet transform, which extracts contrast of pixel pairs in radial 'wedges', not all of these statistical measures are appropriate. Previous research in multi-resolution texture analysis [2] and [3] suggests the following statistics: energy, entropy, mean, and standard deviation. This article introduces the use of several combinations of those descriptors and presents a comprehensive analysis determining the optimal texture descriptors for the curvelet transform as applied to CT scans. Results indicate that using a feature vector containing all four curvelet-based texture descriptors significantly improves the wavelet-based and ridgeletbased classification algorithm.

\section{METHODOLOGY}

The texture classification algorithm proposed in this article consists of four main steps: segmentation of regions of interest, application of the discrete curvelet transform, extraction of texture features, and creation of a classifier.

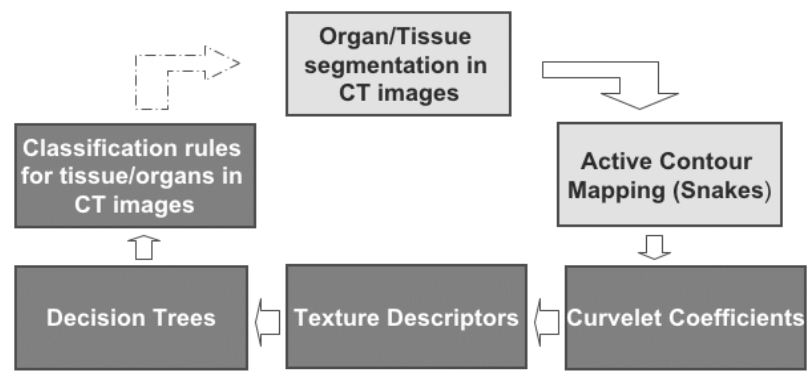

Figure 1: Methodology Diagram

Our tests were conducted on 3D data extracted from two normal chest and abdomen CT studies from Northwestern Memorial Hospital. The data consisted of 340 2D DICOM consecutives slices, each slice being 512 x 512 and having 12-bit grey level resolution. The segmentation process generated 140 Backbone slices, 52 Heart, 58 Liver, 54 Kidney, and 40 Spleen. Curvelets, like wavelets, are extremely sensitive to contrast in the grey level intensity, the segmented images need further processing. In order to effectively use curvelet-based texture descriptors, it was necessary to eliminate all background pixels to avoid mistaking the edge between the artificial background and the tissue as a texture feature. Each slice was therefore further cropped, and only square $32 \times 32$ sub-images fully contained in the interior of the segmented area were generated. This size was chosen since the digital curvelet requires a $2^{\mathrm{n}}$ square image; this is discussed in more detail in [8]. The cropping of the images resulted in 2,091 slices of "pure" single-organ tissue (363 Backbone, 446 Heart, 506 Liver, 411 Kidney, and 364 Spleen).

The texture features used in the algorithm are derived from the Discrete Curvelet Transform, introduced by Candes and Donoho in [8]. This is a discretization of their continuous curvelet transform [7], which uses a "wrapping" algorithm. The transform consists of four steps: application of a 2-dimensional fast Fourier transform of the image, formation of a product of scale and angle windows, wrapping this product around the origin, and application of a 2-dimensional inverse fast Fourier transform. The approximate scales and orientations are supported by a generic 'wedge'.

The discrete curvelet transform can be calculated to various resolutions or scales and angles. Two parameters are involved in the digital implementation of the curvelet transform: number of resolutions and number of angles at the coarsest level. For our images of 32 x 32, maximum resolution extraction was three levels of resolution, and 16 angles were found to be ideal.

Several features were then calculated on the curvelet coefficients. The most common statistics calculated on wavelets are mean and standard deviation. The limited literature on both curvelet-based and ridgelet-based descriptors also suggests the use of a combination of 
mean, standard deviation, energy, and entropy signals (see for example the author's previous work [3]). One of the goals of this research is to identify the most effective texture descriptors for medical images. Mean, standard deviation, energy, and entropy were investigated and their discriminatory power compared. Each of these first order statistics were calculated for each curvelet matrix based on each scale and orientation. The following four feature vectors were investigated: Energy signatures (Eng), Entropy signatures (Ent), Energy and Entropy signatures (EE), and Energy, Entropy, Mean, and Standard Deviation signatures (EEMSD). Each of these feature vectors was computed for three levels of resolution and each radial 'wedge' (16 angles) yielding 18, 36, and 72 descriptors respectively. Our results indicate that curvelet-based texture extraction algorithm outperforms both the ridgelet-based and the wavelet-based algorithms presented in [2], [3].

The classification step was carried out using a decision tree classifier based on the Classification and Regression Tree (C\&RT) approach [1]. A decision tree predicts the class of an object from values of predictor variables or texture descriptors. The most relevant texture descriptors are found for each specific organ, and based on those selected descriptors, a set of decision rules are generated. These set of rules are then used for the classification of the each region. To evaluate the performance of each classifier; specificity, sensitivity, precision, accuracy rates are then calculated from each of the misclassification matrices [see Table 1].

\begin{tabular}{|l|l|}
\hline Measure & Definition \\
\hline Sensitivity & True Positive / Total Positive \\
\hline Specificity & True Negative / Total Negatives \\
\hline Precision & True Positive / (True Positive + False Positives) \\
\hline Accuracy & (True Positives + True Negatives) / Total Sample \\
\hline
\end{tabular}

Table 1: Performance Measures

In the medical domain, the most important performance measures are both specificity and sensitivity. Optimally one would want both high specificity and high sensitivity measures. However, theoretically these two measures should have a negative correlation. Since accuracy reflects both the sensitivity and specificity in relation to each other, this descriptor was selected to determine the overall correctness of the classifier.

\section{RESULTS}

Curvelet-based features yield accuracy rates between 97 and $98 \%$, which significantly improves accuracy rates for Ridgelet-based features [3] and Wavelet-based features [2] [shown in Table 6]. Results indicate that for medical images, a combination of Energy, Entropy, Mean and Standard Deviation (EEMSD) signatures is the most effective descriptors for curvelets. Using 16 angles with EEMSD was found optimal yielding 97 - 98\% accuracy, in comparison with the EEMSD feature vector containing 12 angles and 20 angles, resulting in $94-98 \%$ and 81 $87 \%$ accuracy respectively. The Energy, Entropy, Mean, and Standard Deviation signatures (EEMSD) were in the range 97 - 98\% accuracy [see Table 1], and clearly outperformed all other feature vectors. Energy descriptors were significantly lower than all other feature vectors, with accuracy rates between 80 - 90\%.

Table 2 shows EEMSD as having accuracy rates between 97 - 98\%, which was higher than both Entropy (Ent) signatures (in the 91 - 97\% range) and Energy and Entropy (EE) signatures (in the 91 - 98\%). EEMSD had significantly higher rates for heart, liver, kidney, and spleen increasing the accuracy by approximately $4-6 \%$. Sensitivity rates [see Table 4] were between 89 - 99\% for EEMSD, 75 - $94 \%$ for EE, and 74 - 92\%. EEMSD was significantly higher in all organs. Specificity rates [see Table 5] were much closer in comparison yielding results between 98 - 99\% for EEMSD, 92 - 99\% for EE and 92 98\% for Ent. Overall, the addition of Energy descriptors did not significantly raise the performance of classification; this can be explained by the poor results obtained by using Energy signatures alone. The addition of Mean and Standard Deviation signatures significantly increased performance among all organs.

Previous studies were carried out using wavelet-based and ridgelet-based texture descriptors [2], [3]. The results also show that the curvelet-based texture features using EEMSD outperform all wavelet-based and ridgelet-based descriptors. Accuracy rates [see Table 6] for waveletbased texture descriptors range between 85 - 93\%, while ridgelet-based descriptors were $93-98 \%$, and curveletbased accuracy rates were in the 97 - 98\% range. Overall, the curvelet-based descriptors had significantly higher performance measures, with accuracy rates approximately 5 - 12\% higher than any other feature set for all individual organs. This was expected due to the fact that the curvelet transform is able to capture multi-directional features, as opposed to the wavelet transform which focuses mainly on horizontal, vertical, and diagonal features, which are not dominant in medical CT scan images.

The curvelet-based algorithm has significantly improved upon previous texture classification algorithms described in [1] - [3]. The algorithm presented in this article is able to classify normal tissues in CT scans with high accuracy rates. Although, theoretically curvelets are more complex, there are no significant differences in the time performance. The authors intend to explore the use of 3D curvelet descriptors as well as using curvelet-based descriptors to classify anomalies in the various tissues found in CT scans.

\section{REFERENCES}

[1] Xu, D., Lee, J., Raicu, D.S., Furst, J.D., \& Channin, D. Texture Classification of Normal Tissues in Computed 
Tomography. The 2005 Annual Meeting of the Society for Computer Applications in Radiology. (2005).

[2] Semler, L., Dettori, L., \& Furst, J. Wavelet-Based Texture Classification of Tissues in Computed Tomography. Proceedings of the 18th IEEE International Symposium on Computer-Based Medical Systems, 265-270. (2005).

[3] Semler, L., \& Dettori, L. A Comparison of Wavelet-Based and Ridgelet-Based Texture Classification of Tissues in Computed Tomography. Proceedings of International Conference on Computer Vision Theory and Applications, (2006).

[4] Kara, B. \& Watsuji, N. Using Wavelets for Texture Classification. IJCI Proceedings of International Conference on Signal Processing, ISN 1304-2386, (2003).

[5] Do, M.N., \& Vetterli, M. The Finite Ridgelet Transform for Image Representation. IEEE Transactions on Image Processing, 12, 16 - 28. (2003).

[6] Starck, J.L., Donoho, D.L., \& Candes, E.J. Astronomical

\begin{tabular}{|l|ccc|}
\hline Accuracy & Ent & EE & EEMSD \\
\hline Backbone & 97.5 & 98.2 & 98.9 \\
Heart & 91.8 & 91.9 & 97.1 \\
\hline Kidney & 91.7 & 92.2 & 97.6 \\
Liver & 94.2 & 94.4 & 98.0 \\
\hline Spleen & 94.2 & 92.5 & 97.6 \\
Average & 93.5 & 93.8 & 97.9 \\
\hline
\end{tabular}

Table 2: Accuracy rates comparison

\begin{tabular}{|l|ccc|}
\hline Sensitivity & Ent & EE & EEMSD \\
\hline Backbone & 92.7 & 94.2 & 99.4 \\
Heart & 74.0 & 75.5 & 89.7 \\
\hline Kidney & 89.1 & 92.3 & 96.0 \\
Liver & 89.2 & 81.1 & 95.9 \\
\hline Spleen & 80.0 & 78.4 & 91.8 \\
Average & 83.6 & 84.3 & 94.6 \\
\hline
\end{tabular}

Table 4: Sensitivity rates comparison
Image Representation by the Curvelet Transform. Astronomy \&Astrophysics, 398, 785-800. (1999).

[7] Candes, E. J. \& Donoho, D. L., Curvelets, Multi-resolution Representation, and Scaling Laws, Wavelet Applications in Signal and Image Processing VIII, SPIE 4119, (2000).

[8] Candes, E., Demanet, L., Donoho, D., \& Ying, L. "Fast Discrete Curvelet Transforms. ”(2005).

[9] Haralick, R.M. \& Shapiro, L.G. (1992). Computer and Robot Vision. Addison-Wesley Publishing Co., (1992).

[10] A. Kurani, D.H. Xu, J.D. Furst, D.S. Raicu . "Cooccurrence matrices for volumetric data", The 7th IASTED International Conference on Computer Graphics and Imaging CGIM 2004, Kauai, Hawaii, USA, (2004).

[11] D. H. Xu, A. Kurani, J. D. Furst, \& D. S. Raicu, "Runlength encoding for volumetric texture", The 4th IASTED International Conference on Visualization, Imaging, and Image Processing - VIIP 2004, Marbella, Spain, (2004).

\begin{tabular}{|l|ccc|}
\hline Precision & Ent & EE & EEMSD \\
\hline Backbone & 94.4 & 96.3 & 95.3 \\
Heart & 82.8 & 82.0 & 95.5 \\
\hline Kidney & 77.4 & 77.1 & 93.5 \\
Liver & 88.6 & 90.6 & 94.3 \\
\hline Spleen & 77.7 & 79.7 & 94.9 \\
Average & 84.2 & 85.1 & 94.7 \\
\hline
\end{tabular}

Table 3: Precision rates comparison

\begin{tabular}{|l|ccc|}
\hline Specificity & Ent & EE & EEMSD \\
\hline Backbone & 98.7 & 99.1 & 98.8 \\
Heart & 96.2 & 95.9 & 99.0 \\
\hline Kidney & 92.5 & 92.1 & 98.1 \\
Liver & 97.3 & 97.8 & 98.5 \\
\hline Spleen & 94.9 & 95.6 & 98.9 \\
Average & 95.9 & 96.1 & 98.7 \\
\hline
\end{tabular}

Table 5: Specificity rates comparison

\begin{tabular}{|l|l|cccc|}
\hline Organ & Descriptor & Sensitivity & Specificity & Precision & Accuracy \\
\hline \multirow{3}{*}{ Backbone } & Wavelet & 82.6 & 96.1 & 82.6 & 93.7 \\
& Ridgelet & 91.5 & 99.3 & 96.8 & 98.0 \\
& Curvelet & 99.4 & 98.8 & 95.3 & 98.9 \\
\hline \multirow{3}{*}{ Heart } & Wavelet & 59.0 & 92.1 & 67.0 & 85.0 \\
& Ridgelet & 82.5 & 97.5 & 88.5 & 94.6 \\
& Curvelet & 89.7 & 99.0 & 95.5 & 97.1 \\
\hline \multirow{3}{*}{ Kidney } & Wavelet & 77.7 & 91.4 & 69.9 & 88.6 \\
& Ridgelet & 95.4 & 93.3 & 82.0 & 93.8 \\
& Curvelet & 96.0 & 98.1 & 93.5 & 97.6 \\
\hline \multirow{3}{*}{ Liver } & Wavelet & 87.3 & 94.4 & 82.6 & 92.8 \\
& Ridgelet & 86.9 & 95.9 & 84.4 & 94.0 \\
& Curvelet & 95.9 & 98.5 & 94.3 & 98.0 \\
\hline \multirow{3}{*}{ Spleen } & Wavelet & 65.5 & 94.3 & 69.7 & 89.5 \\
& Ridgelet & 76.9 & 97.6 & 88.0 & 93.8 \\
\hline \multirow{2}{*}{ Average } & Curvelet & 91.8 & 98.9 & 94.9 & 97.6 \\
& Wavelet & 74.4 & 93.7 & 74.4 & 89.9 \\
& Ridgelet & 86.6 & 96.7 & 88.0 & 94.8 \\
& Curvelet & 94.6 & 98.7 & 94.7 & 97.9 \\
\hline
\end{tabular}

Table 6: Comparison of the best wavelet, ridgelet (Entropy), and curvelet-based (EEMSD) descriptors 